\title{
Unusual Locked Trigger Finger Due to Tophaceous Infiltration of Wrist Flexor Tendon
}

\author{
William Hau Wuei Huang*, Juzaily Fekry Leong, Shalimar Abdullah and Jamari Sapuan \\ Department of Orthopaedic and Traumatology, University Kebangsaan Malaysia, Kuala Lumpur, Malaysia
}

Received: December 12, 2017; Published: December 15, 2017

*Corresponding author: William Hau Wuei Huang, Department of Orthopaedic and Traumatology, Faculty of Medicine, University Kebangsaan Malaysia, Kuala Lumpur, Malaysia, Tel: +60 126760192; Email: william_hau@rocketmail.com

\begin{abstract}
Tophi can present as a first sign of hyperuricemia but reports on the involvement of the flexor tendons of the hand is rare. Although tuberculous tenosynovitis is a more common etiology for swelling over the wrist, gout must be among the differentials irrespective of hyperuricemic status. Because of its low frequency, gouty involvement of the flexor tendons is not often considered in the differential diagnosis of tenosynovitis. We report a rare case in which intratendinous tophaceous gout was found within the flexor digitorumsuperficialis tendon at the wrist causing patient to have trigger finger like symptoms.
\end{abstract}

Keywords: Tophaceous Gout; Trigger finger; Tenosynovectomy

\section{Introduction}

Gout is an inflammatory arthritis caused by cellular deposition of monosodium urate crystal [1]. Tophi are chalky, gritty accumulations of monosodium urate crystals that build up in soft tissue in an untreated gouty joint [1]. The peak incidence of gout is between the ages of 30-50 with the prevalence increasing with age [1]. Gout is five times more common in men [1]. Tophi can present as a first sign of hyperuricemia but reports on the involvement of the flexor tendons of the hand is rare [2]. Although tuberculous tenosynovitis is a more common etiology for swelling over the wrist, gout must be among the differentials irrespective of hyperuricemic status. It can present clinically as tendon rupture, nerve compression, or digital stiffness and can be complicated with infection1. Because of its low frequency, gouty involvement of the flexor tendons is not often considered in the differential diagnosis of tenosynovitis [2]. We report a rare case in which intratendinoustophaceous gout was found within the flexor digitorum superficialis tendon at the wrist causing patient to have trigger finger like symptoms.

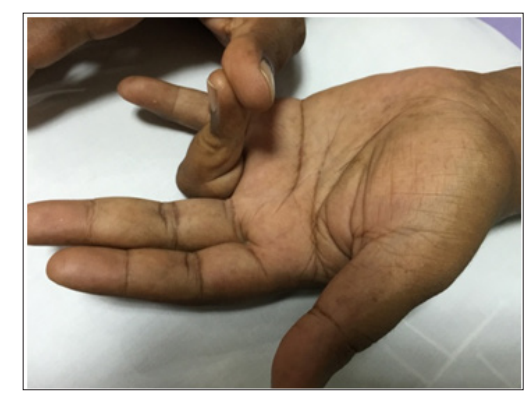

Figure 1: (Volar view): The ring finger in flexed position.

\section{Case Report}

A 37-year-old male presented to our orthopaedic clinic with inability to extend his left ring finger. He previously diagnosed with left trigger ring finger and had surgical treatment to relieve the symptoms at another hospital centre 8 months before coming to our hospital. Post-operatively he was still unable to extend his finger (Figure 1). On physical examination, there was a swelling of $2 \times 2 \mathrm{~cm}$ found over the volar aspect of the forearm just proximal to the flexor retinaculum when the patient flexes his fingers. However on finger extension, the mass disappears. Due to prolong inability to extend his left ring finger he had developed a flexion contracture. Our working diagnosis was incomplete released of left 4th finger A1 pulley, TB tenosynovitis, followed by soft tissue neoplasmand subsequently surgical exploration was performed. We proceed with surgical exploration over the previous surgical scar over the A1 pulley of left ring finger. We noted that the A1 pulley has been completely released. However his finger was still in the flexed position. We decided to do an extended carpal tunnel incision to explore the mass proximal to flexor retinaculum. We noted whitish chalky infiltration of the Flexor Digitorum Superficialis (FDS) tendon, synovial adhesion to other tendons and hypertrophy of the flexor tendon (Figure $2 \& 3$ ). Synovectomy and excision of the chalky infiltration of the FDS tendon was performed. Histo-pathological evaluation confirmed the diagnosis of gout. Post-operatively, patient was able to extend his left ring finger but with some degree of stiffness but after 2 months of intensive physiotherapy he regained full range of movement to that finger. 


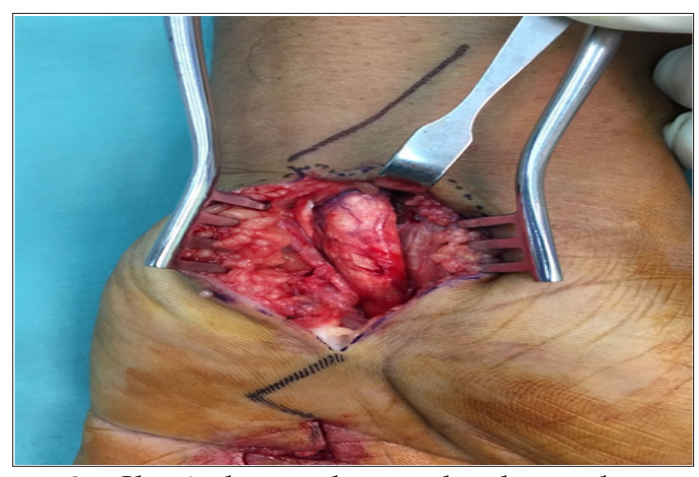

Figure 2: Classical carpal tunnel release done-noted whitish chalky infiltration of the tendon of the FDS, synovial adhesion to other tendons and hypertrophy of the flexor tendon.

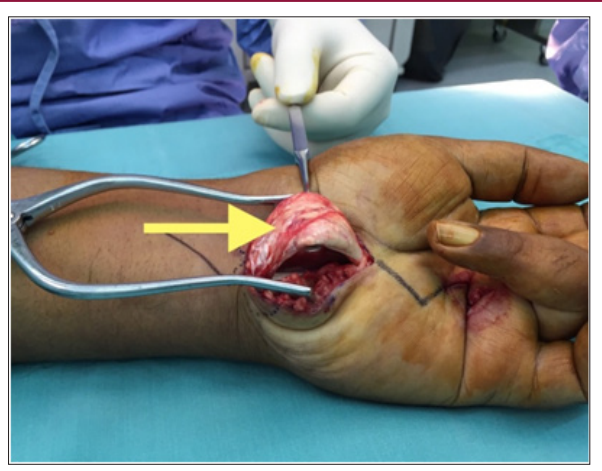

Figure 3: The arrow shows tophaceous gout in the FDP of the left ring finger.

\section{Discussion}

Gout is a disorder of purine metabolism that predisposes to hyperuricaemia, leading to monosodium urate crystal depositions in joints [3]. The underlying metabolic disorder is hyperuricemia [4]. The most common primary cause is renal under excretion (90\%) [5]. Some patients suffer from enzyme defects, which lead to overproduction of uric acid [5]. Dietary causes like high consumption of alcohol or purine-rich foods may also lead to hyperuricemia [5]. Other causes are rare genetic disorders, medical disorders (metabolic syndrome, renal failure, hemolytic anemia), and medication use [5]. Approximately $10 \%$ of patients with elevated blood levels of uric acid develop gout at some point in their life [5]. In patients in whom the disease has been neglected tophaceous destruction of musculoskeletal structures may be helped by carefully selected surgical procedures [4]. The pathological process involvesdeposition of urates and cause destruction to the skin, ligament, tendon and cartilage [4]. This process will cause inflammatory response at the site of involvement and cause symptoms to patient. Lesions may be encapsulated in bursae and subcutaneous tissue and infiltrative in skin, tendon, and bone [4].

The exact trigger of an acute attack of gout is poorly understood however predictors for the development of gout in hyperuricemic individuals have been identified. These include: increasing uric acid level, alcohol consumption, hypertension, use of diuretic drugs (thiazides and loop diuretics), increased body mass index, and family history of gout [3]. In addition to joints, uric acid crystals are reported to deposit in soft tissues such as tendons, median nerve, bursae and intrinsic muscles. Gouty arthritis of the wrist is uncommon although gout itself is the most common inflammatory arthritis in older patients [3]. Gout at the wrist as the initial appearance of the condition occurs between 0.8 to $2 \%$ of all gout cases. Gout patients who are not treated have a $19-30 \%$ chance of developing gout in the wrist [1] during their lifetime. Gouty tenosynovitis can induce flexion contracture of the digits by involvement of the flexor tendons at the wrist, as in our patient, or at the digital canal [2]. Gouty tophi presenting as mass are uncommon and often mistaken for a neoplasm [1].

These nodules may not be recognized as tophi because the clinical diagnosis of gout in many instances is not straightforward [3]. In the past reports, all intratendinous infiltrations of tophaceous gout occurred at the wrist and existed with carpal tunnel syndrome [1]. We present an uncommon and unusual case of gout in the flexor tendon of the forearm which occurred in isolation in a patient with no prior medical history of the recorded disease. Measurement of serum uric acid level in chronic tophaceous gout may or may not be conclusive of hyperuricemia as some patients with diabetes or even alcoholics can have normal to lower levels. Surgical intervention like tenotomy or teno synovectomy are required to debulktophaceous deposits, improve smooth gliding of tendon and decompress nerves but primarily medical management to treat gout remains the gold standard [2]. Short-term outcomes are consistently good but the risk of rupture or recurrence remains if medical control is not achieved.

\section{Conclusion}

This is an uncommon and unusual case of tophaceous infiltration of the flexor digitorumsuperficialis of the ring finger. This case demonstrates several issues that clinicians should keep in mind when assessing patients with a history of gout. Early diagnosis based on a high index of suspicion is paramount to the initiation of proper surgical management.

\section{References}

1. Kumar R, Sahni VK, Jauhar S (2015) Finger Flexion Contracture: First Manifestation of Gout. Journal of Orthopaedic Case Reports April-June 5(2): 66-68.

2. Hernandez Cortes P, Caba M, Gomez Sanchez R, Gomez Morales M (2011) Digital Flexion Contracture and Severe Carpal Tunnel Syndrome Due to Tophaceus Infiltration of Wrist Flexor Tendon: First Manifestation of Gout. Orthopaedics 34(11): 797-799.

3. Craig LJ, Paula JS (2007) An unusual case of gout in the wrist: the importance of monitoring medication dosage and interaction. A case report. Chiropr Osteo pat 15: 16.

4. Larmon WA, L Kurtz JF (1958) The Surgical Management of Chronic Tophaceous Gout. J Bone Joint Surg Am 40(4): 743-772.

5. Mittag F, Wuenschel M (2011) Giant Gouty Tophi of the Hand and Wrist. Orthopaedics 34(11): 790-792. 


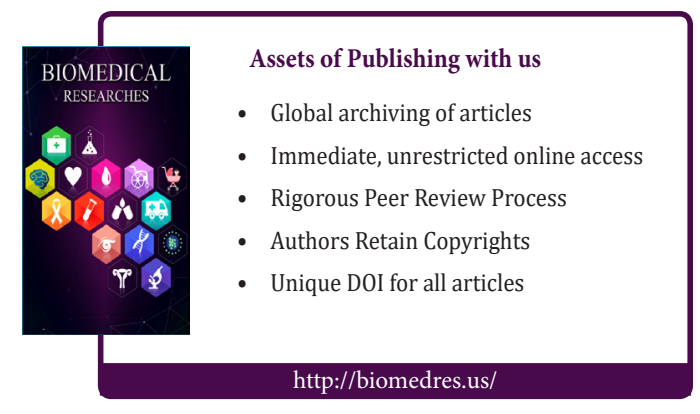

